\title{
ITINERARIO DE ENSEÑANZA PARA EL ÁLGEBRA TEMPRANA
}

\author{
TEACHING ITINERARY FOR EARLY ALGEBRA
}

Ángel Alsina, angel.alsina@udg.edu

Universidad de Girona, Girona, España

\section{RESUMEN}

En este artículo se presenta el Enfoque de los Itinerarios de Enseñanza de las Matemáticas, un enfoque que trata de ser respetuoso con las necesidades reales de los estudiantes para aprender matemáticas. En la primera parte se presenta la fundamentación del enfoque, que se sustenta en tres pilares interrelacionados: la perspectiva sociocultural del aprendizaje humano, el modelo de formación realista-reflexivo y la educación matemática realista; en la segunda parte se describe el enfoque, que se refiere a una secuencia de enseñanza intencionada que contempla tres niveles: 1) enseñanza en contextos informales (el entorno inmediato, los materiales manipulativos y los juegos); 2) enseñanza en contextos intermedios (recursos literarios y tecnológicos), y 3) enseñanza en contextos formales (recursos gráficos); finalmente, en la tercera parte se ejemplifica dicho enfoque con un itinerario de enseñanza del álgebra temprana para estudiantes de 3 a 12 años. Se concluye que la implementación de este enfoque requiere un amplio dominio de conocimientos didáctico-disciplinares, lo que implica un esfuerzo importante por parte de todos los agentes implicados en la formación del profesorado para que así, todo aquel profesional preocupado por mejorar su práctica docente y adaptarla a las exigencias del siglo $\mathrm{XXI}$, pueda tener acceso a estos conocimientos.

\section{PALABRAS CLAVE:}

Didáctica de las matemáticas; itinerario de enseñanza;

álgebra temprana.

\begin{abstract}
This article presents the Mathematics Teaching Itinerary Approach, which tries to be respectful of the students' real needs to learn mathematics. The first part presents the foundation of the approach, which is based on three interrelated pillars: the Sociocultural Perspective of Human Learning, the Realistic-Reflective Training Model and Realistic Mathematical Education; The second part describes the approach, which refers to a sequence of intentional teaching that includes three levels: 1) teaching in informal contexts (the immediate environment, manipulatives and games); 2) teaching in intermediate contexts (literary and technological resources); 3) teaching in formal contexts (graphic resources); Finally, this approach is exemplified in the third part with an itinerary for teaching early algebra for students aged 3 to 12 years. It is concluded that the implementation of this approach requires a broad mastery of didactic-disciplinary knowledge, which requires an important effort from all agents involved in teacher training so all professionals concerned with improving their teaching practice and adapting it to the S. XXI requirements may have access to this knowledge.
\end{abstract}

\section{KEYWORDS:}

Mathematics education; teaching itinerary; early

algebra. 


\section{Introducción}

Todos los que de alguna forma u otra estamos vinculados a la Didáctica de las Matemáticas (maestros, estudiantes para maestro, formadores de maestros, investigadores, etc.) sabemos que el libro de texto es un recurso que ha ejercido una influencia notable en la educación matemática que reciben los estudiantes en las escuelas. Este hecho es la consecuencia de muchos factores de distinta naturaleza en los que aquí no vamos a profundizar, pero sí enumerar: la concepción tradicional de la enseñanza de las matemáticas; el recubrimiento curricular "garantizado"; la medición del aprendizaje en el contexto familiar con base en el volumen de páginas completadas; los intereses económicos de las editoriales, entre otros.

La toma de consciencia de estos factores debería desencadenar una profunda reflexión acerca del papel de este recurso en las aulas. Dicha reflexión debería sustentarse también en los resultados de numerosos estudios centrados en el rendimiento de los estudiantes, que han puesto de manifiesto que el uso exclusivo del libro de texto conlleva descontextualización de los aprendizajes $y$, en consecuencia, genera desmotivación en los estudiantes (Alsina, 2010; Olmos y Alsina, 2010); o bien otros estudios centrados en el análisis de los libros de texto de matemáticas en distintas etapas educativas y en relación a distintos contenidos, que evidencian falencias y errores conceptuales (Azcarate y Serradó, 2006; Gómez, 2001; Vásquez y Alsina, 2015, 2017; entre muchos otros).

Para subsanar esta realidad y transformarla, diversos ámbitos de investigación en educación matemática y sus respectivas agendas de investigación (Alsina, 2019a; Llinares, 2008) han puesto el foco tanto en el análisis didáctico como en la construcción del conocimiento matemático.

En este artículo nos centraremos en la agenda de investigación sobre "análisis de contextos de enseñanza y/o recursos didácticos: situaciones de vida cotidiana, materiales manipulativos, juegos, recursos tecnológicos y gráficos", que corresponde a una de las agendas del ámbito de investigación sobre análisis didáctico (Alsina, 2019, p. 188). En concreto, presentaremos un enfoque de enseñanza que intenta ser más respetuoso con las necesidades reales para aprender matemáticas: el Enfoque de los Itinerarios de Enseñanza de las Matemáticas. Desde este prisma, el artículo se organiza en tres secciones: en la primera se presenta la fundamentación de este enfoque; en la segunda se describe qué es un itinerario de enseñanza de las matemáticas; $y$, finalmente, en la tercera se ejemplifica un itinerario de enseñanza para el álgebra temprana de 3 a 12 años.

\section{Fundamentación del Enfoque de los Itinerarios de Enseñanza de las Matemáticas}

Tal como se muestra en la figura 1, este enfoque se fundamenta en tres pilares interrelacionados: la perspectiva sociocultural del aprendizaje humano (Vygostsky, 1978); el modelo de formación realistareflexivo (Melief, Tigchelaar y Korthagen, 2010; Tigchelaar, Melief, Van Rijswijk y Korthagen, 2010), adaptado a la formación del profesorado de matemáticas (Alsina, 2019b); y la Educación Matemática Realista (EMR) de Freudenthal (1991).

De la perspectiva sociocultural del aprendizaje humano, que se fundamenta en las aportaciones de Vygotsky (1978) y en las reinterpretaciones de su obra que han hecho otros autores tanto desde el ámbito de la psicología del aprendizaje (Ivic, 1994; Wertsch, 1985, 1991) como desde el campo de la educación matemática (Lerman, 2000, 2001; Schmittau, 2004; entre otros), interesa principalmente destacar que la educación se concibe como un fenómeno social y cultural que es posible gracias a la interacción, la negociación y el diálogo, junto con la idea de que el pensamiento intelectual depende de la construcción autorregulada del conocimiento, que va de un proceso interpsicológico a un proceso intrapsicológico (Alsina y Domingo, 2010).

Del modelo de formación realista-reflexivo, que ha sido desarrollado en la Universidad de Utrecht (Países Bajos) por Korthagen (2001), Melief et al. (2010), entre otros, por un lado interesa destacar que pretende, a través de la reflexión sistemática, impulsar la integración de la persona con sus experiencias personales y como aprendices, con sus conocimientos teóricos y con sus representaciones sobre lo que es enseñar y aprender, razón por la cual se usa el término "realista-reflexivo" (Esteve y Alsina, 2010). Desde este prisma, se asume que los educadores deberían llegar a conocer muchas maneras de actuar y ejercitarlas en la práctica, es decir, deberían disponer de criterios para saber cuándo, qué y por qué algo es conveniente y reflexionar sobre ello sistemáticamente (Korthagen, 2001); y por otro lado interesa la aplicación de este modelo en la formación de maestros de Matemáticas. En este sentido, Alsina (2019b) presenta desde una perspectiva analítica, interpretativa y crítica los resultados de diversos estudios que muestran los avances en investigación sobre la formación de maestros de Matemáticas desde el modelo realista-reflexivo. En concreto, se presentan cuatro estudios sobre diseño, aplicación y análisis de episodios desde este modelo de 
formación que incluyen un ciclo formativo; diversos recursos y estrategias didácticas para promover la deconstrucción de los conocimientos cotidianos (CC) de los maestros de Matemáticas que pueden ser un obstáculo para su desarrollo profesional; la identificación de las marcas de autorregulación que permiten deconstruir CC y coconstruir y reconstruir conocimientos profesionales (CP); y, como síntesis de los estudios preliminares, la descripción de un modelo transformador de la competencia profesional de los futuros maestros de Matemáticas.

Finalmente, de la EMR (Freudenthal, 1991), que nace en el Instituto para el Desarrollo de la Educación Matemática de la Universidad de Utrecht, hoy conocido como Freudenthal Institute for Science and Mathematics Education, interesan sobre todo sus seis principios: de actividad, de realidad, de reinvención guiada, de niveles, de interacción y de interconexión. En síntesis, en la EMR se utilizan situaciones de la vida cotidiana o problemas contextualizados como punto de partida para aprender matemáticas. Progresivamente, estas situaciones son matematizadas a través de modelos, mediadores entre lo abstracto y lo concreto, para formar relaciones más formales y estructuras abstractas (Heuvel-Panhuizen, 2002). Además, se apoya en la interacción en el aula entre los estudiantes y entre el profesor y los estudiantes (Fauzan, Plomp y Slettenhaar, 2002), junto con la idea de que a los estudiantes se les debería dar la oportunidad de reinventar las matemáticas bajo la guía de un adulto en lugar de intentar transmitirles una matemática preconstruida (De Corte, Greer y Verschaffel, 1996).

A partir de este conjunto de planteamientos se ha diseñado el Enfoque de los Itinerarios de Enseñanza de las Matemáticas, que se describe en la próxima sección.

\section{El Enfoque de los Itinerarios de Enseñanza de las Matemáticas}

Hace ya varios años, y con el propósito de buscar maneras ajustadas a las necesidades reales de los niños y niñas tanto para aprender matemáticas como para aprender a usarlas de forma comprensiva y eficaz en todas las situaciones -escolares y no escolaresen las que dichos conocimientos son necesarios, Alsina (2010) planteó un diagrama piramidal en el que se comunicaba de forma sencilla y muy visual el tipo de contextos necesarios para desarrollar el pensamiento matemático y su "frecuencia de uso" más recomendable, en función de la posición que ocupa cada contexto: de más o menos frecuencia desde la base hacia la cúspide (figura 1). En este diagrama piramidal, más conocido como Pirámide de la Educación Matemática al hacer un símil con la Pirámide de la Alimentación, no se descartaba ningún recurso, sino que solo se pretendía informar sobre la conveniencia de restringir algunos de ellos a un uso ocasional y, por eso, se consideró que podía ser una herramienta útil para el profesorado preocupado por hacer de su metodología una garantía de educación matemática.

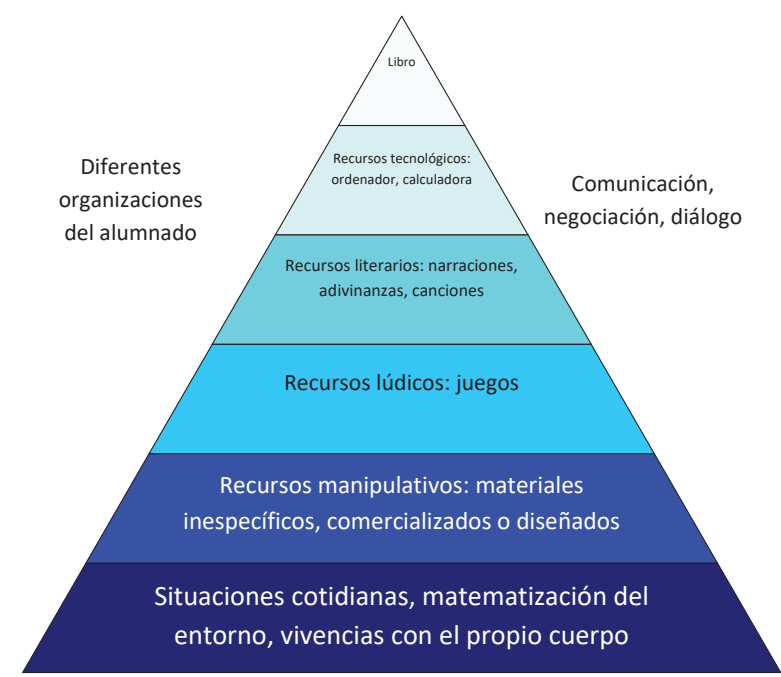

Figura 1. Pirámide de la Educación Matemática Fuente: Alsina (2010, p. 14)

En la base se situaban los contextos que necesitan todos los estudiantes y que, por lo tanto, se podrían y deberían "consumir" diariamente para aprender matemáticas: las situaciones problemáticas y los retos que surgen en la vida cotidiana de cada día, la observación y el análisis de los elementos matemáticos del entorno, la manipulación con materiales diversos y los juegos, entendidos como la resolución de situaciones problemáticas. Después seguían los que deben "tomarse" alternativamente varias veces a la semana, como los recursos literarios y los recursos tecnológicos. Y, por último, en la cúspide, se ubicaban los recursos que deberían usarse de forma ocasional, concretamente los libros de texto, por las razones que ya se han expuesto en la introducción.

Con los años, este planteamiento ha evolucionado hacia el Enfoque de los Itinerarios de Enseñanza (Alsina, 2019c), asumiendo que la palabra "itinerario" se refiere a una secuencia de enseñanza intencionada que contempla tres fases:

1. Enseñanza en contextos informales: la enseñanza del contenido matemático se inicia 
en situaciones reales o realistas de los niños, como por ejemplo su entorno inmediato, o bien materiales manipulativos y juegos, en los que el conocimiento de la situación y las estrategias se utilizan en el contexto de la situación misma, apoyándose en los conocimientos informales, el sentido común y la experiencia.

2. Enseñanza en contextos intermedios: la enseñanza del contenido prosigue en contextos que hacen de puente entre los contextos reales o realistas de la fase previa y los contextos formales de la fase posterior, como por ejemplo algunos recursos literarios (cuentos y canciones) y tecnológicos (applets, robots educativos programables, etc.), que a través de la exploración y la reflexión conducen a la esquematización y generalización progresiva del conocimiento matemático.

3. Enseñanza en contextos formales: la enseñanza del contenido finaliza en contextos gráficos y simbólicos, como por ejemplo el lápiz y el papel, en los que se trabaja la representación y formalización del conocimiento matemático con procedimientos y notaciones convencionales para completar de esta forma el aprendizaje desde lo concreto hasta lo simbólico.

Este enfoque, pues, se aleja de una visión de la enseñanza de las matemáticas basada en la repetición y la práctica de ejercicios que presentan los libros de texto como principales estrategias didácticas para "aprender" matemáticas, y en su lugar, plantea que es necesario fomentar la comprensión más que la mera memorización, la actividad heurística más que la pura ejercitación, o el pensamiento matemático crítico más que la simple repetición.

La comprensión, la actividad heurística y el pensamiento matemático crítico, como indica Alsina (2019c), son algunos de los principales pilares sobre los que se sustenta la educación matemática del siglo XXI. Necesitamos, más que nunca, formar a ciudadanos que comprendan en profundidad el conocimiento matemático para que puedan aplicarlo en todas las situaciones a lo largo de la vida en las que, de una forma u otra, están implicadas las matemáticas. Necesitamos también formar a ciudadanos que descubran por sí mismos las ideas matemáticas a través de una planificación y gestión adecuadas de las prácticas matemáticas basadas en la resolución de problemas, el razonamiento y la prueba, la comunicación, la argumentación, las conexiones, la modelización y la representación, más que transmitirles un conocimiento matemático ya construido previamente. Y, por supuesto, necesitamos formar a ciudadanos que procesen e interpreten críticamente la gran cantidad de datos que recibimos constantemente a través de diferentes medios (prensa escrita, televisión, internet, etc.). En definitiva, necesitamos formar a ciudadanos que sean capaces de plantear problemas y preguntas vitales con claridad y precisión; que evalúen información relevante; que lleguen a conclusiones y soluciones, probándolas con criterios relevantes, y que se comuniquen con eficacia a la hora de idear soluciones.

Esta visión contemporánea de la educación matemática requiere, primero, de un amplio dominio profesional de los conocimientos matemáticos a enseñar, puesto que no se puede enseñar bien lo que no se sabe y, segundo, un amplio dominio profesional acerca de las formas de enseñar dichos conocimientos, puesto que los estudiantes de hoy no tienen las mismas necesidades para aprender matemáticas que los estudiantes de años atrás, por lo que no tiene ningún sentido enseñar lo mismo que hace décadas y menos aún enseñarlo de la misma forma. Por esta razón, en la última sección de este artículo se presenta una ejemplificación de un itinerario de enseñanza focalizado en un bloque de contenidos relativamente nuevo en los planteamientos curriculares de las primeras edades: el álgebra temprana.

\section{Itinerario de enseñanza del álgebra temprana}

En esta sección se describe, en primer lugar, la presencia del álgebra en el currículo de matemáticas de las primeras edades, y en segundo lugar se presenta un ejemplo de itinerario para una enseñanza eficaz del álgebra temprana, ajustada a las necesidades reales de aprendizaje de los estudiantes.

\subsection{El álgebra en el currículo de matemáticas de las primeras edades}

El álgebra es un bloque de contenidos matemáticos que tradicionalmente se ha asociado a niveles de escolarización posteriores, para tratar conocimientos vinculados a la generalización y al simbolismo principalmente. Sin embargo, hay que considerar que existe también una larga tradición de otros conocimientos de naturaleza algebraica previos a la generalización y el simbolismo en edades inferiores, ya desde la etapa de Educación Infantil y, por supuesto, también en la etapa de Educación Primaria: los distintos tipos de relaciones (clasificaciones y ordenaciones, por ejemplo), los patrones o bien los cambios forman parte del cuerpo de conocimientos algebraicos que se aprenden desde edades tempranas, y que de hecho 
son la base para la adquisición de otros conocimientos matemáticos.

Autores de prestigio como Montessori, Piaget o Dienes situaron estos conocimientos dentro de "la lógica", "la lógica matemática" o bien "el razonamiento lógicomatemático". Sus planteamientos tuvieron una gran repercusión en la escuela, y lograron introducir la idea de que en las primeras edades estos conocimientos se adquirían a partir de situaciones concretas con materiales manipulativos, pero no los vincularon explícitamente al álgebra, sino que los consideraban parte de la educación sensorial o bien de la inteligencia lógico-matemática, respectivamente (Alsina, 2019d).

Sin embargo, los currículos de matemáticas de las primeras edades más avanzados han empezado a sustituir paulatinamente estos términos por el término "álgebra temprana" (Early Algebra) al considerarse como una puerta de entrada a las matemáticas superiores, entre otras cosas porque aporta un lenguaje enriquecido capaz de crear la base con que se enseñan las matemáticas (Stacey y Chick, 2004). Así, por ejemplo, en las orientaciones curriculares de Estados Unidos, desde que el National Council of Teachers of Mathematics (NCTM, 2000) avanzara el trabajo sistemático del álgebra temprana a partir de los 3 años, se han ido concretando los puntos focales curriculares en los que se debería centrar el proceso de enseñanza y aprendizaje del álgebra temprana desde Pre-Kindergarten hasta Kindergarten (3 a 6 años aproximadamente). Estos puntos se centran en ordenar y clasificar objetos atendiendo a determinadas propiedades, reconocer y ampliar patrones tanto de secuencias sonoras como numéricas y analizar el comportamiento de los patrones utilizando representaciones concretas, pictóricas y verbales, entre otros aspectos (NCTM, 2006). En el Nurturing Early Learners (NEL), que es la versión revisada del currículo vigente del Ministerio de Educación de la República de Singapur, se indica que uno de los objetivos para los estudiantes de 4 a 6 años es "reconocer y usar relaciones y patrones simples" (Ministry of Education of Singapore, 2013, p. 22). En la guía para docentes que despliega de manera más detallada este objetivo, se sugiere crear patrones repetidos usando objetos, palabras, dibujos, símbolos o acciones, así como reconocer y extender patrones simples. En Australia, el Australian Curriculum, Assessment and Reporting Authority (ACARA, 2015) aboga por un desarrollo del sentido de número, del orden, la secuencia, el patrón y la posición haciendo uso del contexto del estudiante. Concretamente, en relación con el álgebra y los patrones, plantea que los estudiantes de 4 años ordenen, clasifiquen objetos familiares, expliquen la base de la clasificación ejecutada, copien, creen y extiendan patrones con objetos, dibujos o material manipulable, además de reforzar la capacidad de observar e identificar los patrones naturales del entorno. Y en Nueva Zelanda, uno de los intereses y capacidades crecientes de los estudiantes de las primeras edades que se expone en Te Whāriki: Early Childhood Curriculum (Ministry of Education of New Zealand, 2017) es, precisamente, ser capaz de desarrollar conceptos matemáticos tempranos como la clasificación y la percepción de patrones que inciten a la indagación, exploración y evaluación de todo aquello que resulte inesperado. De esta forma, se asegura establecer una base permeable que permita a los estudiantes de etapas posteriores explorar el uso de los patrones y las relaciones que se pueden establecer con aspectos de cantidad, conjuntos de datos, espacio y tiempo.

\subsection{Ejemplificación de un itinerario de enseñanza del álgebra temprana de 3 a 12 años}

El planteamiento de un itinerario de enseñanza para desarrollar el pensamiento algebraico en las primeras edades, como se ha señalado, responde al firme convencimiento de que para garantizar un aprendizaje sólido es imprescindible llevar a cabo una enseñanza eficaz, fundamentada en un amplio conocimiento de contextos, recursos y estrategias didácticas. Junto con estos conocimientos, es necesario también disponer de criterios para secuenciar la enseñanza de manera que sea respetuosa con las necesidades y las posibilidades de los estudiantes (Alsina, 2019c, 2019d).

Con base en estas consideraciones, se propone una secuenciación que se inicia en los contextos de enseñanza-aprendizaje del álgebra temprana más reales y realistas y culmina en los contextos más abstractos, de acuerdo con el Principio de Niveles planteado por Freudenthal (1991), que sugiere que la matematización es progresiva, nace totalmente ligada al contexto que la requiere, a partir del cual se esquematiza, se abstrae, se sale de la situación misma, se generaliza, se formaliza... todo progresivamente, paso a paso en esos distintos niveles de comprensión.

4.2.1. Contextos informales: situaciones de vida cotidiana, materiales manipulativos y juego para la enseñanza del álgebra temprana de 3 a 12 años

En este primer nivel del itinerario para la enseñanza del álgebra temprana se consideran, como se ha señalado, los contextos de enseñanza-aprendizaje más reales o realistas en las mentes de los estudiantes. El criterio general es que los estudiantes deberían empezar a identificar conocimientos algebraicos en su entorno y, a través de sus experiencias en la escuela 
con materiales manipulativos y juegos, deberían llegar a tener más habilidad para descubrir dichos conocimientos y avanzar de esta forma en habilidades como la generalización.

Desde esta perspectiva, a continuación, se describe una experiencia de vida cotidiana y se presenta una selección de recursos lúdico-manipulativos para enseñar contenidos de álgebra temprana, teniendo en cuenta que en la planificación y gestión de estas actividades se deberían considerar los procesos - habilidades matemáticas de resolución de problemas, razonamiento y prueba, comunicación, argumentación, conexiones, modelización y representación.

4.2.1.1. Una experiencia a partir de un contexto real: una visita por la ciudad

La experiencia que se describe, denominada "Redescubriendo la calle Mayor de Palencia con ojos matemáticos" (Alsina, Novo y Moreno, 2016), consiste en observar patrones en el entorno.

Lugar de implementación: Escuela Pública "Sofía Tartilán" (Palencia, España)

Nivel: 5-6 años

Maestra responsable de la implementación: Asunción Moreno

Asesoramiento pedagógico: Ángel Alsina y $\mathrm{M}^{\mathrm{a}}$ Luisa Novo

Contenidos matemáticos:

- Concepto de patrón como secuencia que se repite.

- Identificación de patrones, de dos o más elementos, y de dos o más criterios.

- Realización de seriaciones con objetos, siguiendo un determinado patrón.

- Representación de patrones.

Descripción de la actividad: En primer lugar, en la clase los estudiantes hacen seriaciones siguiendo un determinado patrón de repetición con material no estructurado (tapones de diversos tamaños, formas y colores) y con material estructurado (bloques lógicos, tarjetas de conceptos gráficos, formas figurativas o abstractas...). A continuación, manipulan libremente regletas, hacen seriaciones y las representan en un papel cuadriculado. Los estudiantes van describiendo los patrones: "regleta blanca, regleta roja tumbada, regleta blanca, regleta roja tumbada..."; "regleta roja de pie, regleta verde tumbada, regleta roja de pie, regleta verde tumbada..."; "regleta verde para abajo, regleta blanca tumbada...".
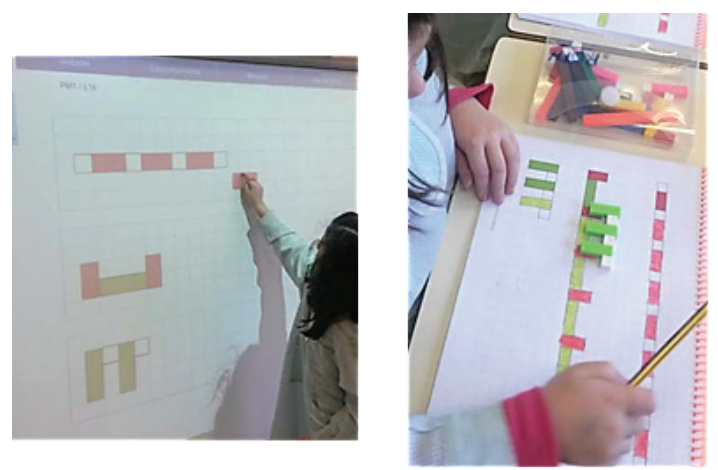

Figura 2. Seriaciones con regletas en la pizarra digital y en el papel cuadriculado

Fuente: Alsina et al. (2016, p. 14)

Ya en la calle Mayor de Palencia pueden evidenciar patrones en elementos urbanísticos. Les resulta más fácil descubrir patrones en áreas cercanas a ellos, como por ejemplo el suelo... áreas que abarcan fácilmente por estar en su entorno corporal. Y si, además, hay un contraste marcado entre los elementos, lo perciben mejor. Es el caso de un embaldosado:

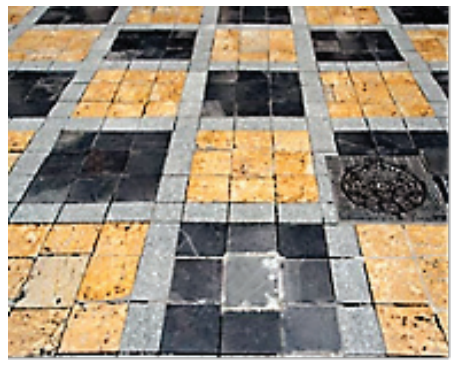

Figura 3. Series con baldosas Fuente: Alsina et al. (2016, p. 14)

- Niños: ¡Hay una serie! Sí, cuadrado negro, cuadrado amarillo, cuadrado negro, cuadrado amarillo...

Sobre la pizarra digital se trabajan algunas fotografías, donde reconocen la calle Mayor. Se plantea el reto de que descubran qué patrones hay:

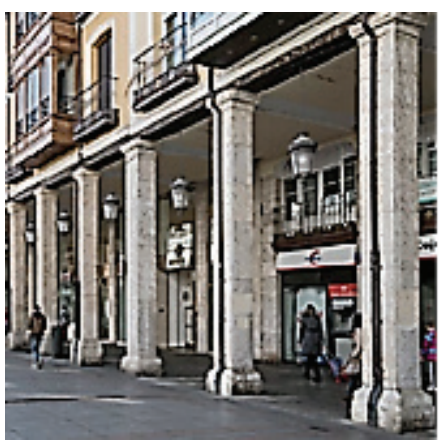

Figura 4. Columnas en la calle Fuente: Alsina et al. (2016, p. 15) 
- Niño: Me sé una serie yo

- Niña: Y yo otra.

- Niña: Balcón, balcón y ¿esto?

- Maestra: Eso es un mirador.

- Niña: Balcón, balcón, mirador, balcón, balcón, mirador..

- Niño: Yo sé otra... Con lo que sujeta...

- Maestra: ¿Cómo se llama?

- Niño: ¿Columna?

- Maestra: Sí. Y sigue...

- Niño: Columna, no hay nada, columna, no hay nada, columna, no hay nada..

- Maestra: Mira un poco hacia arriba, ¿qué ves?

- Niño: ¿Una farola?

- Maestra: Es un farol porque no se apoya en el suelo. - Niño: Columna, farol, columna, farol, columna, farol...

Finalmente, ante la fotografía del portón rápidamente un niño descubre un patrón:

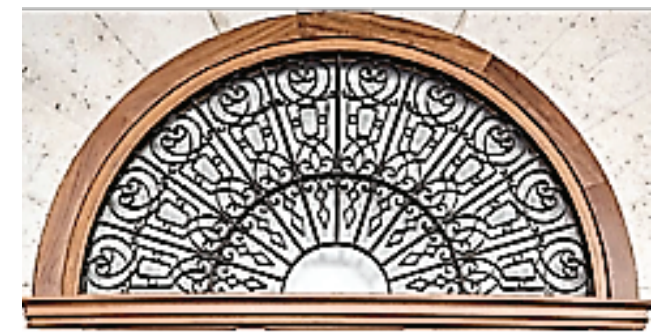

Figura 5. Portón

Fuente: Alsina et al. (2016, p. 15)

- Niño: Tiene una serie que me la sé. Y señala: Dibujo, raya, dibujo, raya, dibujo, raya...

\subsubsection{Materiales manipulativos y juegos}

Se presenta una selección de materiales lúdicomanipulativos para trabajar contenidos de álgebra temprana de forma concreta. Los materiales se han seleccionado con el propósito de que sirvan, por una parte, para trabajar algunos conocimientos indispensables para facilitar la introducción al álgebra temprana, como por ejemplo materiales lógicos estructurados para trabajar las agrupaciones de elementos o bien relaciones de equivalencia (clasificaciones) y correspondencias por distintos criterios, tanto cualitativos como cuantitativos. Por otra, se pone mucho énfasis en los materiales que sirven para trabajar patrones numéricos y geométricos, tanto de repetición como de crecimiento (y decrecimiento), y se han seleccionado también algunos materiales manipulativos y juegos para el análisis de cambios, en un sentido amplio: situaciones en las que los cambios se mantienen constantes (como por ejemplo el dominó de las diferencias, donde se determina previamente el número de cambios entre una pieza y la otra, y esta cantidad de cambios se mantiene constante) o bien situaciones en las que la tasa de cambio aumenta o disminuye, como por ejemplo analizar cuántos triángulos se generan con diferentes cantidades de garbanzos, desde 3 hasta la cantidad que se desee (Cardet, 2009).

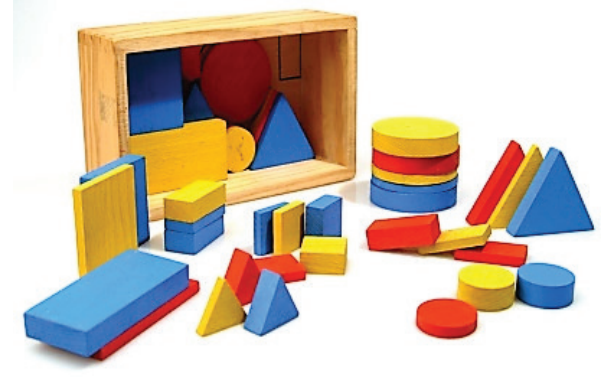

Bloques lógicos de Dienes para realizar agrupaciones, clasificaciones, correspondencias cualitativas, etc. (según el color, la forma, el tamaño o el grosor).

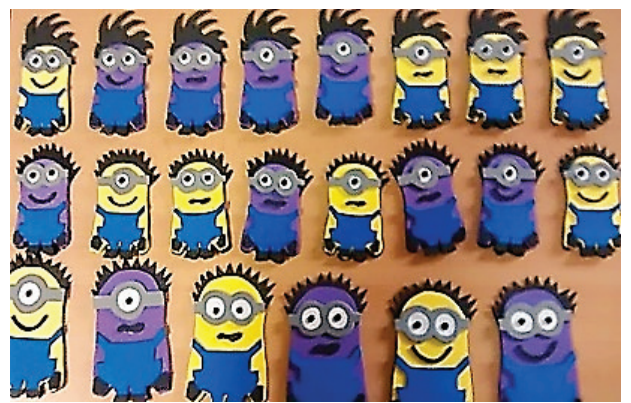

Otros materiales lógicos para realizar también agrupaciones, clasificaciones, correspondencias cualitativas, etc. (según el color, la cantidad de ojos, el estado de ánimo, etc.).

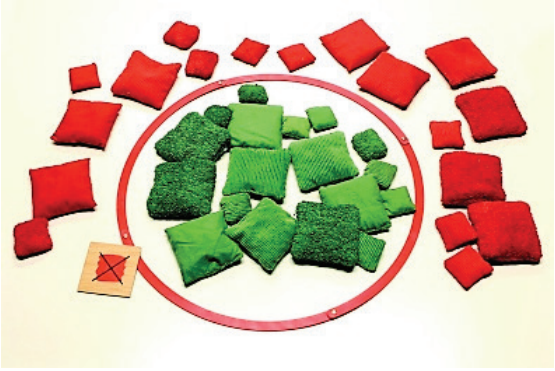

Ejemplo de agrupación a partir de etiquetas negativas. 


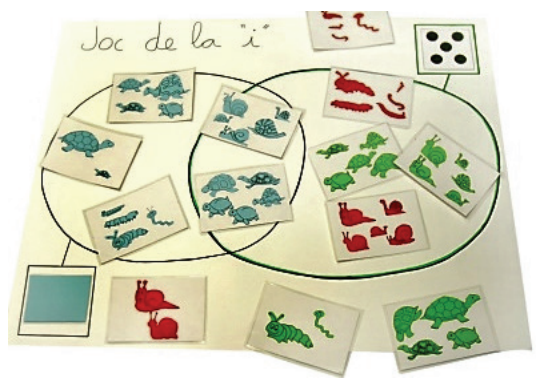

Ejemplo de agrupación con dos cualidades: "ser de color azul" y "tener cinco elementos". Se generan cuatro espacios: "los azules", "los que hay cinco", "los que son azules y hay cinco" y "los que no son azules ni hay cinco".

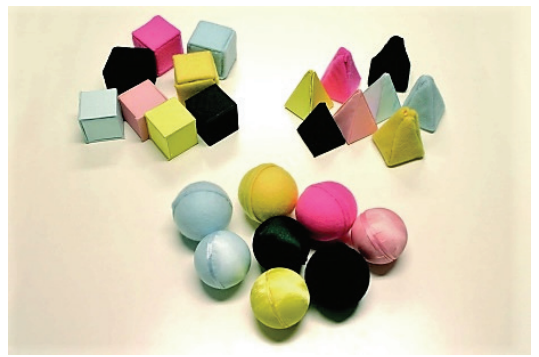

Ejemplo de clasificación (relación de equivalencia) por un criterio de forma: cubos, pirámides y esferas.

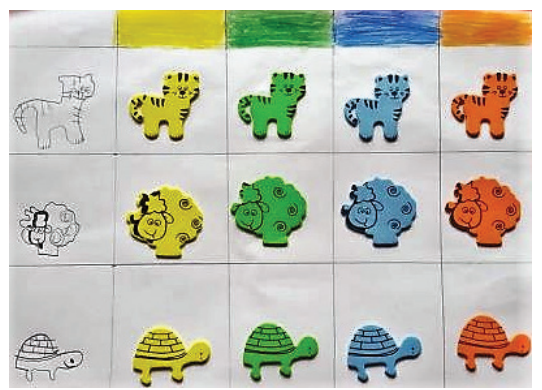

Ejemplo de correspondencia cualitativa a partir de un cuadro de doble entrada (producto cartesiano)

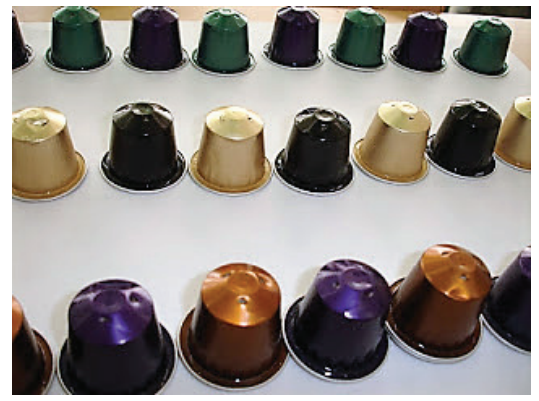

Cápsulas de café para construir series (patrón de repetición $A B$ ).

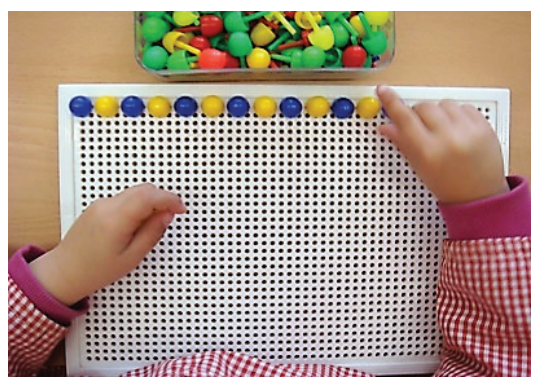

Pinchos de colores para construir series (patrón de repetición AB).

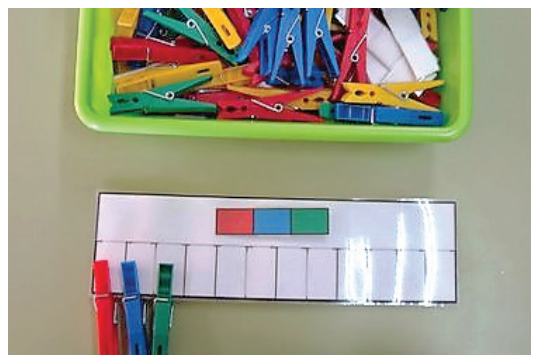

Pinzas para construir series (patrón de repetición $A B C$ ).

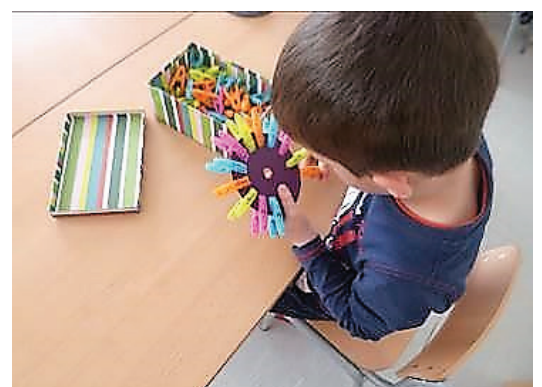

Pinzas para construir series circulares (patrón de repetición $A B C$ ).

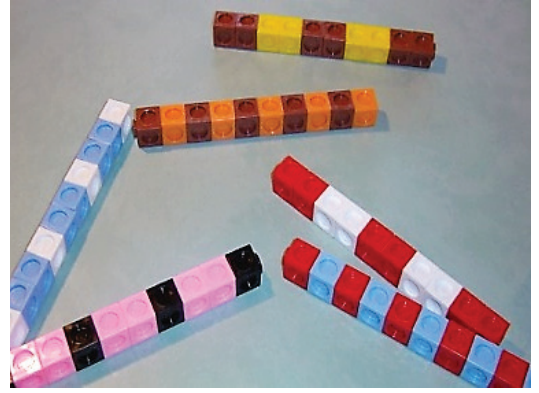

Policubos para construir series y clasificarlas según el patrón de repetición ( $A B, A B B, A A B B)$. 


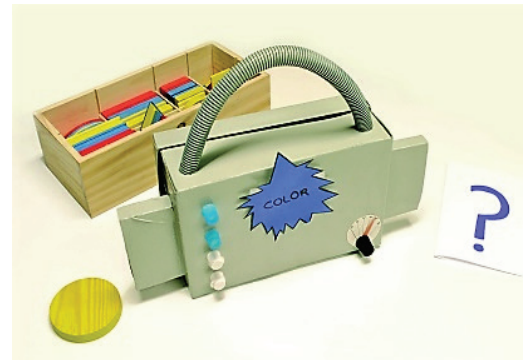

Máquina de cambios constantes: entra un círculo amarillo, el operador indica el cambio y deben pensar qué pieza sale.

Figura 6. Algunos recursos lúdico-manipulativos para estudiantes de 3 a 8 años

Fuente: Alsina (2019c, p. 129)

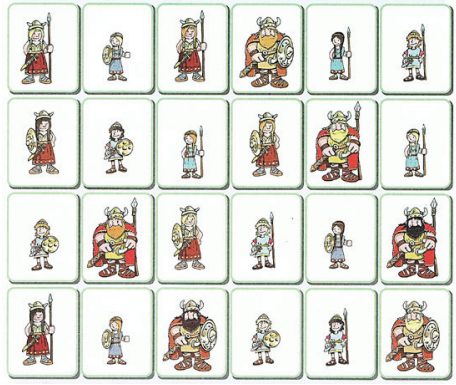

Cartas de vikingos (Alsina, 2004) para realizar agrupaciones, clasificaciones, correspondencias, etc. Tienen las cualidades siguientes: cuatro miembros de la familia; tres colores del pelo y dos instrumentos (escudo o lanza).

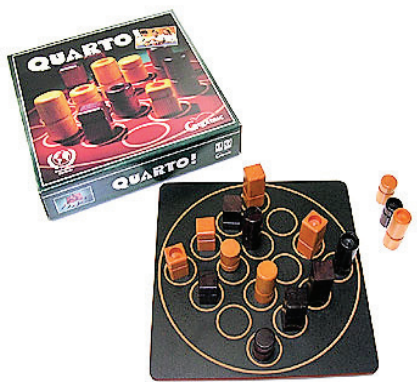

Juego "Quarto" para realizar agrupaciones, clasificaciones, correspondencias cualitativas, etc. Se trata de torres con las cualidades siguientes: dos colores, dos alturas, dos formas, vacío o lleno.

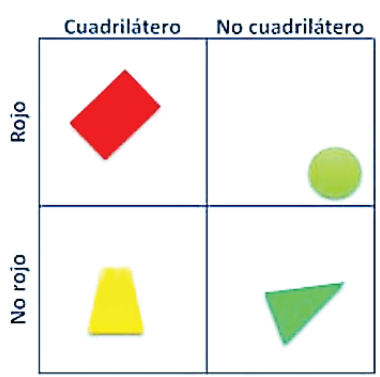

Figuras geométricas y diagramas de Caroll para representar dos o más conjuntos

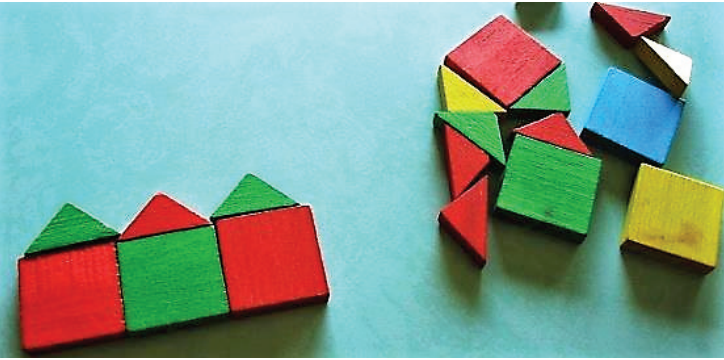

Piezas para construir series según un patrón de repetición más complejo y representarlo (por ejemplo: aBbA, donde las piezas verdes se representan con "a/A" según si son triángulos o cuadrados, respectivamente, y las piezas rojas se representan con "b/B" siguiendo el mismo criterio).

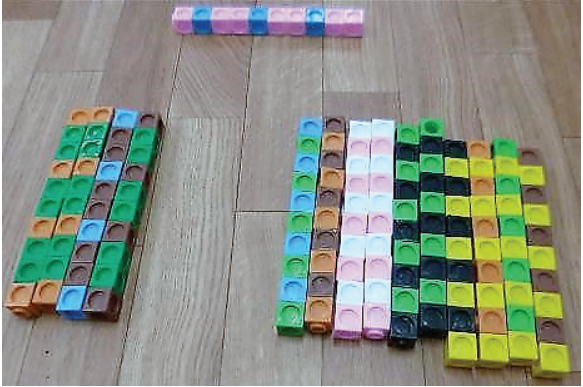

Policubos para construir series y clasificarlas según el patrón de repetición ( $A B B$ y $A B$, respectivamente), para luego analizar su regularidad mediante tablas y construir una primera idea intuitiva de función afín en los números naturales.
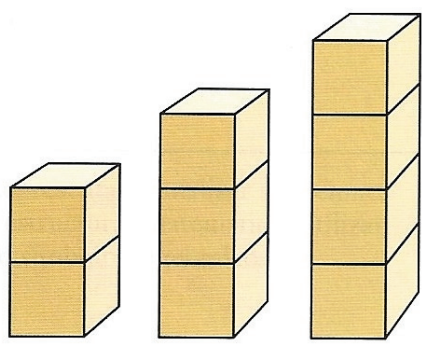

Cubos de madera para construir y analizar series según un patrón de crecimiento: observar cómo aumenta la altura a medida que se van añadiendo cubos.

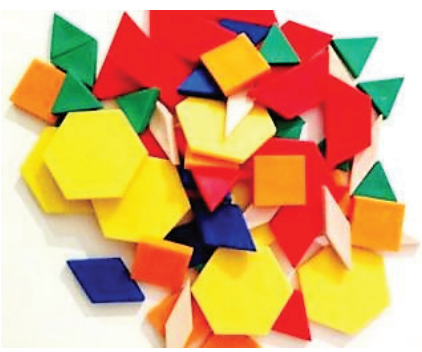

Geomosaico para realizar patrones geométricos 


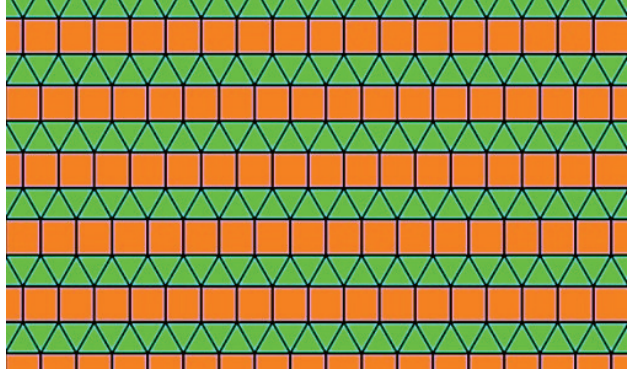

Ejemplo de mosaico (triangular alargado) a partir de un patrón con triángulos verdes y cuadrados naranjas del geomosaico.

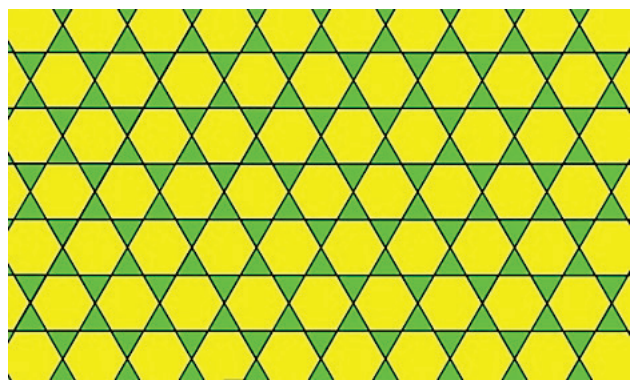

Ejemplo de mosaico (trihexagonal chato) a partir de un patrón con hexágonos amarillos y triángulos verdes del geomosaico.

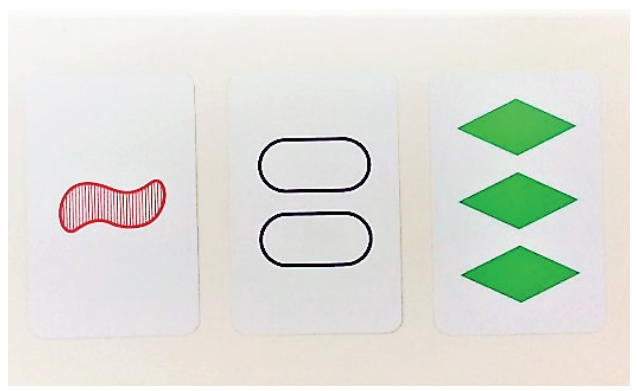

Observación de cambios con las cartas SET: tres cartas forman un SET si, respecto a cada una de las cuatro características (forma, color, número y fondo), evaluadas una a una, las tres cartas son iguales o las tres son diferentes.

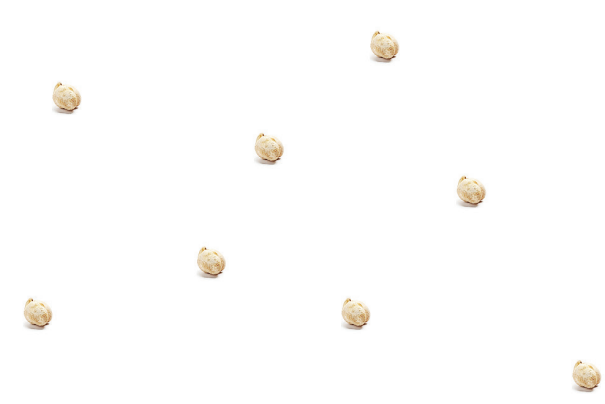

Garbanzos para analizar situaciones con tasas de cambio crecientes (Cardet, 2009): analizar cuántos triángulos se generan con 3, 4, 5, 6,

7, 8... garbanzos y generalizar, escribiendo los datos en una tabla y representándolos en un gráfico, como conocimiento precursor de las funciones (es decir, para comprender más adelante lo que representa la pendiente de una recta).

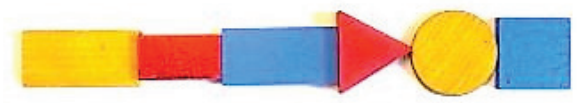

Dominó de las diferencias con tres cambios: se inicia el juego con una pieza y para colocar la siguiente deben cambiar tres cualidades, por ejemplo: rectángulo amarillo, grande y delgado; rectángulo rojo, pequeño y grueso (cambia el color, el tamaño y el grosor); rectángulo azul, grande y delgado (cambia el color, el tamaño y el grosor); etc.

Figura 7. Algunos recursos lúdico-manipulativos para estudiantes de 8 a 12 años Fuente: Alsina (2019c, p. 130-131)

4.2.2. Contextos intermedios: recursos literarios y tecnológicos para la enseñanza del álgebra de 3 a 12 años

Una vez que los estudiantes de las primeras edades hayan tenido la oportunidad de empezar a comprender conocimientos algebraicos ligados a contextos reales o realistas, es recomendable seguir el itinerario de enseñanza de estos conocimientos ofreciendo otros contextos que permitan ir avanzando de manera progresiva en la formalización o institucionalización de los aprendizajes. En este sentido, los recursos literarios y tecnológicos pueden ser contextos ideales 
para hacer de puente entre las matemáticas concretas y las abstractas.

\subsubsection{Recursos literarios}

Para trabajar los contenidos de álgebra temprana durante las primeras edades se pueden utilizar distintos géneros literarios como cuentos y novelas. Estos recursos, en especial los cuentos, habitualmente se han usado para trabajar contenidos de numeración y cálculo, geometría, etc. Sin embargo, como señala Torra (2012), la aportación que hacen los cuentos al aprendizaje de las matemáticas va más lejos de lo que a primera vista se acostumbra a captar y, si bien es cierto que muchos de ellos inciden en aspectos vinculados a la numeración o a la geometría, la verdadera aportación es la presencia de patrones que, como se ha indicado, abren la posibilidad de predecir, de anticipar lo que va a suceder, de buscar regularidades y de generalizar.

En los cuentos se pueden encontrar patrones de repetición, patrones de crecimiento/decrecimiento e incluso estructuras de correspondencia entre elementos de dos conjuntos:

- Los patrones de repetición se encuentran en cuentos clásicos, adecuados principalmente para los estudiantes de los primeros niveles, como por ejemplo "La ratita presumida" en el que la estructura del cuento sigue siempre el mismo patrón, pero con un cambio al final que advierte que, aunque los patrones sirven para predecir o anticipar lo que va a suceder, hay que estar atentos a los cambios y comprobar si las predicciones se cumplen o no.

- Los patrones de crecimiento/decrecimiento se encuentran en cuentos con una estructura que se caracteriza por una sucesión de personajes o eventos que se presentan en orden ascendente o descendente, respectivamente, y no lineal, como en el caso de los patrones de repetición. La suma de estos personajes o eventos es lo que permite, al final, conseguir el objetivo. Es el caso de cuentos como por ejemplo "A qué sabe la luna", que presenta un patrón de crecimiento ya que la estructura se basa en una secuencia de personajes que, juntos, alcanzan la luna; o bien el cuento "Cien hormigas hambrientas", que presenta un patrón de decrecimiento ya que la estructura se basa en una secuencia de sucesos centrados en la descomposición del 100 en sumandos cada vez menores (dos grupos de 50; cuatro grupos de 25; cinco grupos de 20; diez grupos de 10).

- Finalmente, la correspondencia entre elementos de dos conjuntos se encuentra en cuentos cuya estructura se basa en un personaje que tiene una misión por cumplir, se presentan obstáculos y debe buscar estrategias para superarlos, estableciendo así una correspondencia "obstáculo-solución", como por ejemplo "El sastrecillo valiente" o bien otras narraciones de héroes.

Es importante, pues, impulsar que los estudiantes miren más allá del número de personajes que contiene un cuento o bien la trayectoria que hacen los personajes o las formas geométricas que aparecen en él. Hay que fomentar el análisis de la estructura interna y descubrir los patrones, similares en todas las culturas, para que los estudiantes avancen en habilidades tan importantes como predecir y generalizar, que son algunos de los principales objetivos científicos.

A continuación, se describe una posible gestión para facilitar que los estudiantes descubran las regularidades que se esconden en la estructura interna de un cuento:

1. Explicar, visualizar o bien leer todo el cuento o media parte del cuento.

2. Plantear algunas buenas preguntas vinculadas a la estructura (además de cuestiones asociadas a los elementos observables a simple vista), como, por ejemplo: ¿en cuántas partes se divide la historia?; ¿ocurre lo mismo en cada parte?; ¿qué ocurre después de la primera parte?; ¿qué ocurre antes de la tercera parte?; ¿qué piensan que va a ocurrir al final?, ¿por qué?; etc.

3. Finalmente, sugerir a los estudiantes que representen la historia: en los primeros niveles las representaciones pueden ser concretas (con dibujos, por ejemplo) y, progresivamente, se puede invitar a los estudiantes a hacer representaciones más abstractas de los patrones o correspondencias observadas (usando letras, signos, etc.). A modo de ejemplo, en la figura 8 se muestran tres representaciones posibles de los tres tipos de cuentos expuestos:

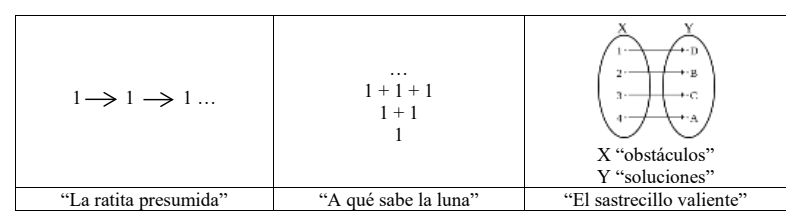

Figura 8. Representaciones de los patrones Fuente: Alsina (2019c, p. 134) 
4.2.2.2. Recursos tecnológicos: calculadora, applets y lenguajes de programación visuales

Estos recursos, asociados a una metodología en la que el estudiante es el protagonista de su aprendizaje, pueden ser muy útiles para facilitar la adquisición de conocimientos algebraicos. A continuación, pues, se ofrece una selección de actividades para seguir trabajando conocimientos algebraicos en este nivel del itinerario didáctico, más próximo ya a la abstracción.

a) Calculadora

En Educación Primaria, más que en Educación Infantil, la calculadora puede ser un buen recurso sobre todo para trabajar conocimientos algebraicos asociados a los patrones. En concreto, los estudiantes pueden explorar patrones numéricos y hacer conjeturas acerca de estos patrones:

- Exploración de patrones numéricos: muchas calculadoras básicas, al teclear un número cualquiera (por ejemplo, el 2), el signo más (+) y a continuación el signo igual $(=)$ de manera repetida, van presentando una progresión o sucesión aritmética: 2, 4, 6, 8, 10, ... En el caso de que la calculadora no tenga esta función, se puede construir sumando repetidamente un mismo dígito: $2+2=4 ; 4+2=6$; ... Lógicamente, la exploración de patrones numéricos con la calculadora se puede complicar con números mayores (por ejemplo, con un patrón $+5 ;+10$; + 25; etc.) o bien también se pueden construir y analizar progresiones o sucesiones geométricas, donde el patrón se basa en un producto. En este caso, al multiplicar un número (por ejemplo, el 2), por un mismo producto de forma repetida (x 2 ), se obtiene una serie de números que progresa de forma geométrica: $2,4,8,16,32, \ldots$

- Realización de conjeturas a partir de patrones: tomando como base las progresiones aritméticas o geométricas construidas, se pueden realizar predicciones, como por ejemplo estimar qué número saldrá al teclear 10 veces el mismo sumando (+ 2) en las progresiones aritméticas o el mismo producto ( $x$ 2) en las progresiones geométricas; así mismo, en los niveles superiores se puede plantear a los estudiantes que generalicen $a$ " $n$ " casos y lo representen algebraicamente: en los dos ejemplos presentados, sería $n+2$ y $2 n$, respectivamente.

\section{b) Applets}

Se pueden encontrar diversos applets muy interesantes para trabajar contenidos algebraicos en las distintas webs:

- NRICH (https://nrich.maths.org)

- Illuminations (https://illuminations.nctm.org)

- Math Playground (http://www.mathplayground. com)

- Biblioteca Nacional de Manipuladores Virtuales

(http://nlvm.usu.edu/es/nav/vlibrary.html)

Aparte de estos recursos, existen también otras páginas web específicas para trabajar conocimientos algebraicos, como por ejemplo Visual Patterns (http:// www.visualpatterns.org), en la que hay centenares de propuestas de patrones visuales para trabajar aspectos numéricos y de introducción al álgebra temprana.

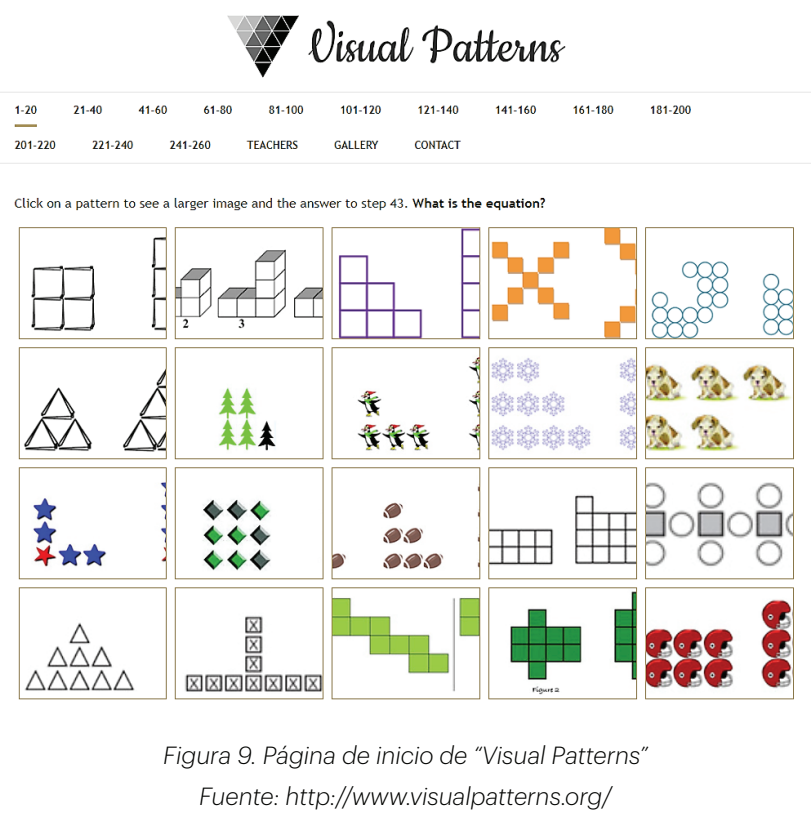

c) Lenguajes de programación visuales

Scratch es un recurso válido tanto para crear proyectos focalizados en conocimientos algebraicos a partir de las funciones de los bloques de este lenguaje de programación, como para practicar contenidos a partir de proyectos previamente diseñados, simplemente buscando el término que interesa: "álgebra", "patrones", "expresiones algebraicas", etc.

En la secuencia de la figura 10, por ejemplo, se muestra una forma sencilla de programar un patrón de repetición a través de un ciclo que consiste en repetir 5 veces la secuencia "mover 50 pasos" y "girar $72^{\circ}$ a la derecha", lo que daría lugar a la construcción de un pentágono regular. 

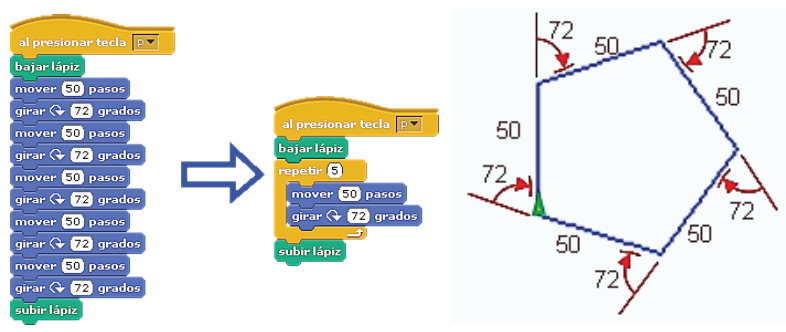

Figura 10. Ejemplo de un patrón de repetición usando la función "repetir" para construir un pentágono regular

Fuente: Alsina (2019c, p. 138)

4.2.3. Contextos formales: recursos gráficos para la enseñanza del álgebra de 3 a 12 años

En la recta final del itinerario de enseñanza, es importante incidir principalmente en la formalización de los conocimientos aprendidos en los contextos preliminares, y más tratándose de conocimientos algebraicos. De hecho, como se ha indicado, una de las finalidades del álgebra es poder expresar mediante lenguaje simbólico las ideas matemáticas, usando expresiones algebraicas que combinan números, signos y letras siguiendo ciertas reglas.

Desde esta perspectiva, además de usar materiales como libros de texto y fichas previamente seleccionados para apoyar este tipo de aprendizaje, se pueden también usar otros recursos que permitan avanzar hacia la formalización. En este apartado destacamos por su novedad, pero sobre todo por su potencial, recursos como: Which One Doesn't Belong? (WODB), creado por el profesor de matemáticas estadounidense Christopher Danielson (https://wodb. $\mathrm{ca} /$ ), que consiste en presentar a los estudiantes varias situaciones distintas, habitualmente cuatro (figura 11), con el objeto de que comuniquen y argumenten cuál de ellas no pertenece al grupo, lo que fomenta la presencia de procesos matemáticos como el razonamiento y la prueba (que se fundamenta en la argumentación y la comprobación) y la comunicación (que se fundamenta en la interacción, la negociación, el diálogo y el uso adecuado de lenguaje matemático).
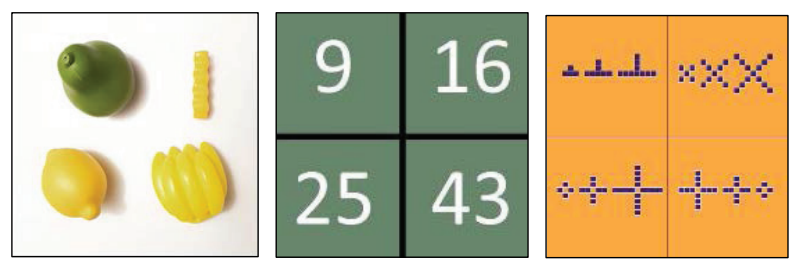

Figura 11. Distintos ejemplos de WODB

Fuente: https://wodb.ca/
Otro recurso parecido es "Same but different", de Looney Math Consulting, disponible en https:// www.samebutdifferentmath.com/about/, en el que se presentan dos situaciones en lugar de cuatro, pero igualmente los estudiantes deben argumentar críticamente en qué son iguales y en qué son diferentes. La sección de álgebra de este recurso presenta parejas mayoritariamente para los niveles superiores de la etapa de Educación Primaria y para etapas posteriores (figura 12):
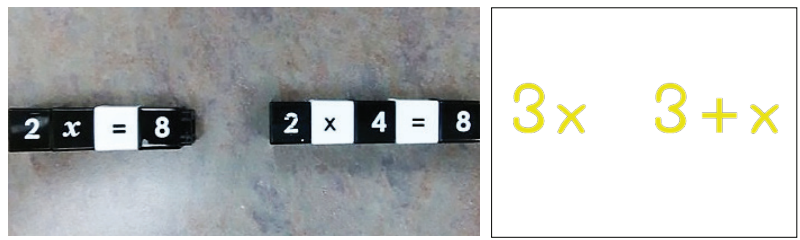

Figura 12. Ejemplos de parejas de la sección de álgebra de Same but different Fuente: Alsina (2019c, p. 139)

\section{Consideraciones finales}

En este artículo se ha fundamentado, descrito y ejemplificado el Enfoque de los Itinerarios de Enseñanza de las Matemáticas (Alsina, 2018, 2019c) que, como se ha argumentado, pretende contribuir a romper con la visión tradicional de la enseñanza de las matemáticas asociada al uso del libro de texto como único recurso, para avanzar hacia una visión más respetuosa con las necesidades reales de los estudiantes de las primeras edades para aprender matemáticas. Para ello, como se indicado, este enfoque considera diversos contextos organizados en tres niveles (contextos informales, intermedios y formales). Además, se plantea la necesidad de llevar a cabo una planificación y gestión de la práctica matemática que se fundamente en la resolución de problemas, el razonamiento y la prueba, la comunicación, la argumentación, las conexiones, la modelización y la representación, y todo ello basado en el principio fundamental de que el conocimiento debe ser siempre construido por el aprendiz a través de la mediación de un adulto, en lugar de transmitir un conocimiento ya creado con anterioridad.

En esta sección final, pues, queremos destacar el hecho de que en el Enfoque de los Itinerarios de Enseñanza se asume que el proceso de enseñanza-aprendizaje de las matemáticas no es una labor sencilla, puesto que implica dominar una serie de conocimientos tanto disciplinares (sobre las matemáticas) como didácticos (sobre formas de enseñar matemáticas y evaluarlas). Ello implica un esfuerzo importante por parte de todos (Administración Educativa, 
instituciones de Educación Superior, organismos y expertos en materia de educación en general y de educación matemática en particular, etc.) para que el profesorado preocupado por mejorar sus prácticas y adaptarlas a las exigencias del siglo XXI pueda tener acceso a estos conocimientos. Este acceso debe promover, por un lado, que puedan reflexionar sobre la necesidad de transformar su práctica docente dejando de focalizar la enseñanza de las matemáticas exclusivamente en un libro de texto- para mejorarla usando diversos contextos de enseñanza y, por otro, que se les facilite la labor aportando recursos que sean factibles de ser usados, ya que como indican Hargreaves, Earl, Moore y Manning (2001), "si el profesor no está dispuesto a hacerlo, no se puede hacer" (p. 128); "si el profesor no sabe cómo hacerlo o a la hora de la verdad no se siente seguro haciéndolo, no se puede hacer" (p. 129); "si un docente no está dispuesto a hacerlo, no se puede hacer" (p. 132), y "si el profesor tiene que hacer demasiadas cosas, no las hará bien" (p. 134). Esperamos que la ejemplificación de un itinerario focalizado en la enseñanza del álgebra temprana haya contribuido a esta labor, e invitamos al lector a consultar otros itinerarios referentes a otros bloques de contenido en Alsina (2019c) y, sobre todo, a lanzarse a diseñar sus propios itinerarios con el propósito de mejorar la formación matemática de los estudiantes de las primeras edades. 


\section{Referencias}

Alsina, Á. (2004). Barrinem? Matemàtiques amb jocs $i$ problemes. Lògica 3. Barcelona: Edicions I'Àlber, S.L.

Alsina, Á. (2010). La "pirámide de la educación matemática", una herramienta para ayudar a desarrollar la competencia matemática. Aula de Innovación Educativa, 189, 12-16.

Alsina, Á. (2018). Seis lecciones de educación matemática en tiempos de cambio: itinerarios didácticos para aprender más y mejor. Padres y Maestros, 376, 13-20.

Alsina, Á. (2019a). La educación matemática infantil en España: ¿qué falta por hacer? Números. Revista de Didáctica de las Matemáticas, 100, 85-108.

Alsina, Á. (2019b). Hacia una formación transformadora de futuros maestros de matemáticas: avances de investigación desde el modelo realista-reflexivo. Unipluriversidad, 19(2), 60-79.

Alsina, Á. (2019c). Itinerarios didácticos para la enseñanza de las matemáticas (6-12 años). Barcelona: Editorial Graó.

Alsina, Á. (2019d). Del razonamientológico-matemático al álgebra temprana en Educación Infantil. Edma 0-6: Educación Matemática en la Infancia, 8(1), 1-19.

Alsina, Á., y Domingo, M. (2010). Idoneidad didáctica de un protocolo sociocultural de enseñanza y aprendizaje de las matemáticas. Revista Latinoamericana de Investigación en Matemática Educativa, 13(1), 7-32.

Alsina, Á., Novo, M. L., y Moreno, A. (2016). Redescubriendo el entorno con ojos matemáticos: Aprendizaje realista de la geometría en Educación Infantil. Edma 0-6: Educación Matemática en la Infancia, 5(1), 1-20.

Australian Curriculum, Assessment and Reporting Authority. (2015). The Australian Curriculum: Mathematics. Recuperado desde http://v7-5. australiancurriculum.edu.au/Curriculum/Overview

Azcarate, P., y Serradó, A. (2006). Tendencias didácticas en los libros de texto de matemáticas para la ESO. Revista de Educación, 340, 341-378.

Cardet, N. (2009). Els cigrons i la matemàtica. Suplement Guixdos, 156, 1-15.
De Corte, E., Greer, B., y Verschaffel, L. (1996): Mathematics Teaching and Learning. En D. Berliner, y C. Calfee (Eds.), Handbook of Educational Psychology (pp. 491-549). Nueva York: Simon \& Schuster Macmillan.

Esteve, O., y Alsina, Á. (2010). Hacia el desarrollo de la competencia profesional del profesorado. En O. Esteve, K. Melief, y Á. Alsina (Eds.), Creando mi profesión. Una propuesta para el desarrollo profesional del profesorado (pp. 7-18). Barcelona: Editorial Octaedro.

Fauzan, A., Plomp, T., y Slettenhaar, D. (2002). Traditional mathematics education vs. realistic mathematics education: Hoping for Changes. En Proceedings of the 3rd International Mathematics Education and Society Conference (pp. 1-4). Copenhagen: Centre for Research in Learning Mathematics.

Freudenthal, H. (1991). Revisiting mathematics education. Dordrectht: Kluwer Academic Publishers.

Gómez, B. (2001). La justificación de la regla de los signos en los libros de texto: ¿por qué menos por menos es más? En P. Gómez, y L. Rico (Eds.), Iniciación a la investigación en didáctica de la matemática. Homenaje al profesor Mauricio Castro (pp. 257-275). Granada: Editorial Universidad de Granada.

Hargreaves, A., Earl, L., Moore, S., y Manning, S. (2001). Aprender a cambiar. La enseñanza más allá de las materias y los niveles. Barcelona: Editorial Octaedro.

Heuvel Panhuizen, M. (2002). Realistic mathematics education as work in progress. En F. L. Lin (Ed.), Common sense in mathematics education. Proceedings of 2001 The Netherlands and Taiwan Conference on Mathematics Education (pp. 1-43). Taiwan: National Taiwan Normal University.

Ivic, I. (1994). Lev Semionovick Vygotsky (18961934). Perspectivas: Revista Internacional de Educación Comparada, 34(3-4), 773-799.

Korthagen, F. A. (2001). Linking practice and theory. The pedagogy of realistic teacher education. Londres: Lawrence Erlbaum Associates.

Lerman, S. (2000). The social turn in mathematics education research. En J. Boaler (Ed.), Multiple perspectives on mathematics teaching and learning (pp. 19-44), Westport, CT: Ablex.

Lerman, S. (2001). The function of discourse in teaching and learning mathematics: a research perspective. Educational Studies in Mathematics, 46(13), 87-113. 
Llinares, S. (2008). Agendas de investigación en Educación Matemática en España. Una aproximación desde "ISI-web of knowledge" y ERIH. En R. Luengo, B. Gómez, M. Camacho, y L. J. Blanco (Eds.), Investigación en Educación Matemática XII (pp. 25-54). Badajoz: SEIEM.

Melief, K., Tigchelaar, A., y Korthagen, K. (2010). Aprender de la práctica. En O. Esteve, K. Melief, y Á. Alsina (Eds.), Creando mi profesión. Una propuesta para el desarrollo profesional del profesorado (pp. 19-38). Barcelona: Octaedro.

National Council of Teachers of Mathematics. (2000). Principles and Standards for School Mathematics. Reston, VA: Autor.

National Council of Teachers of Mathematics. (2006). Curriculum Focal Points for Prekindergarten through Grade 8 Mathematics: a quest for coherence. Reston, V.A.: Autor.

Ministry of Education of New Zealand (2017). Te Whāriki: Early Childhood Curriculum. Wellington: Autor.

Ministry of Education of Singapore. (2013). Nurturing Early Learners: A Curriculum for Kindergartens in Singapore: Numeracy: Volume 6. Singapore: Autor.

Olmos, G., y Alsina, Á. (2010). El uso de cuadernos de actividades para aprender matemáticas en educación infantil. Aula de Infantil, 53, 38-41.

Schmittau, J. (2004). Vygostkian theory and mathematics education: Resolving the conceptualprocedural dichotomy. European Journal of Psychology of Education, 29(1), 19-43.

Stacey, K., y Chick, H. (2004). Solving the problem with algebra. En K. Stacey, H. Chick, y M. Kendal (Eds.), The Future of Teaching and Learning of Algebra. The 12th ICMI Study (pp. 1-20). Boston: Kluwer.

Tigchelaar, A., Melief, K., Van Rijswijk, M., y Korthagen, K. (2010). Elementos de una posible estructura del aprendizaje realista en la formación inicial y permanente del profesorado. En O. Esteve, K. Melief, y Á. Alsina (Eds.), Creando mi profesión. Una propuesta para el desarrollo profesional del profesorado (pp. 3964). Barcelona: Octaedro.

Torra, M. (2012). Patrones matemáticos en los cuentos. Cuadernos de Pedagogía, 421, 56-58.
Treffers, A. (1987). Three Dimensions. A Model of Goal and Theory Description in Mathematics Instruction The Wiskobas Project. Dordrecht: Reidel Publishing Company.

Vásquez, C., y Alsina, Á. (2015). Un modelo para el análisis de objetos matemáticos en libros de texto chilenos: situaciones problemáticas, lenguaje y conceptos sobre probabilidad. Profesorado, Revista de currículum y formación del profesorado, 19(2), 441-462.

Vásquez, C., y Alsina, Á. (2017). Proposiciones, procedimientos y argumentos sobre probabilidad en libros de texto chilenos de educación primaria. Profesorado, Revista de currículum y formación del profesorado, 21(1), 433-457.

Vygotsky, L. S. (1978). Mind in society. The development of higher psychological processes. Cambridge, MA: Harvard University Press.

Wertsch, J. V. (1985). Vygotsky y la formación social de la mente. Barcelona: Paidós.

Wertsch, J. V. (1991). Voces de la mente. Un enfoque sociocultural para el estudio de la acción mediada. Madrid: Aprendizaje Visor. 